\title{
Effectiveness of two methods for mesocercariae of Alaria alata detection in wild boars (Sus scrofa)
}

\author{
Zanda Ozoliṇa*, Gunita Deksne \\ Institute of Food Safety, Animal Health and Environment "BIOR", Lejupes 3, Riga LV-1076, Latvia \\ ${ }^{*}$ Corresponding author, E-mail: zanda.ozolina@bior.lv
}

\begin{abstract}
Over the past decade, frequent detection of trematode Alaria sp. mesocercariae in wild boar muscle samples during official Trichinella inspection has raised concern as a potential risk of human and animal health. In the present study 60 wild boar muscle samples were examined with two methods, Alaria sp. mesocercariae migration technique (AMT) and the official Trichinella inspection method (TIM). The results of the present study shows that AMT had 4.86 (95\% CI: 2.22-10.99) times higher odds of testing positive than with TIM. The cause of low proportion of positive mesocercariae observation using TIM is mainly associated with inappropriate mesh size of the sieve used in comparison to Alaria alata mesocercariae size. In addition, a shortening the AMT procedure time for 30 min is sufficient to obtain only qualitative results for $A$. alata mesocercariae in muscle samples.
\end{abstract}

Key words: Alaria alata, mesocercariae, mesocercariae migration technique, wild boar.

Abbreviations: AMT, Alaria sp. mesocercariae migration technique; TIM, Trichinella inspection method.

\section{Introduction}

Alaria spp. is a trematode with tetraxenous life cycle and is present in all climatic zones (Galaktionov, Dobrovolskij 2003). Genus Alaria include seven species worldwide, however Alaria alata is the only known species of the genus in Europe (Möhl et al. 2009). This trematode infects muscles of paratenic host (e.g. wild boar and human) and does not form a cyst (Möhl et al. 2009). Mesocercariae is pear-shaped, compressed from ventral side and gibbous from dorsal side and it is 0.3 to $0.7 \mathrm{~mm}$ long and 0.141 to $0.282 \mathrm{~mm}$ wide (Skrjabin 1960; Möhl et al. 2009).

In early studies, Alaria sp. specimens regarding their biology were identified by naked eye or with a magnifying glass, later the compression method was used (Riehn et al. 2010). Nowadays A. alata is accidentally found by Trichinella spp. inspection method (TIM) with artificial digestion with a magnetic stirrer (Jakšić et al. 2002; Möhl et al. 2009; Riehn et al. 2010).

Using TIM, the prevalence of $A$. alata mesocercariae in wild boars ranged from 1.8 to $91.0 \%$ in Croatia and $24.0 \%$ in Branderburg, Germany (Jakšić et al. 2002; Möhl et al. 2009). A five year study in France showed prevalence of 0.6\% (Portier et al. 2014). In Estonia, from 2009 to 2011, A. alata was detected in 52 wild boars in total and an increase of positive findings was observed, four cases 2009 and 24 in 2011 (total investigated sample number not known, Estonian Food and Veterinary Laboratory, unpublished data). In Lithuania, mesocercariae prevalence in wild boars was 40.0 and $30.5 \%$ in 2008 and 2011, respectively
(Lithuanian Food and Veterinary Laboratory, unpublished data). In Latvia, a similar study with the routine samples from official Trichinella inspection from 2010 to 2012 were done and overall in 4088 wild boars analyzed A. alata mesocercariae prevalence was 7\% (Esīte et al. 2013).

The differences in infection mode, predilection sites and the size of Trichinella and A. alata suggest that TIM might be not suitable for mesocercariae detection, causing false and inadequate results (Möhl et al. 2009). For several years, a specific and more sensitive detection method for digenetic trematode A. alata is available, the Alaria sp. mesocercariae migration technique (AMT; Riehn et al. 2010). Still this method is not applied for routine examinations. However, studies which used AMT have greater reliability. Studies in Germany using AMT showed A. alata prevalence 11.5\% in wild boars and $33.3 \%$ in raccoons (Riehn et al. 2012; Rentería-Solís et al. 2013), while during similar study in Austria prevalence in wild boars was 6\% (Paulsen et al. 2014).

During the past decade in Europe, increased attention has been paid to $A$. alata occurrence in wild boars. However, due to the scarcity of methodology and data predicting the risk of human alariosis is difficult. Although the main source of infection is considered the consumption of an insufficiently cooked wild boar meat, A. alata is still not considered to be a zoonotic agent in Europe (Möhl et al. 2009; Riehn et al. 2011a; Riehn et al. 2011b, Riehn et al. 2011c). The aims of present study were to define the level of possible false-negative results using TIM and to improve the AMT to shorten as possible performance time. 


\section{Materials and methods}

Overall, in the present study samples from 60 wild boars were collected and analyzed during 2014 from the whole territory of Latvia. Hunters were asked voluntarily to submit wild boar muscle samples. Each sample contained 200 to $350 \mathrm{~g}$ of different types of muscles (diaphragm, tongue and other skeletal muscles) appropriate for Trichinella testing. Only sufficient samples (enough amount of the sample; not frozen before submission to the laboratory; no features of decomposing) were selected for the present study. All samples were stored at $2{ }^{\circ} \mathrm{C}$ until examination, which was performed within $24 \mathrm{~h}$.

Artificial digestion method with magnetic stirrer for Trichinella sp. detection (Regulation EC No 2075/2005) and Alaria sp. migration technique (Riehn et al. 2010) was used to detect Alaria sp. mesocercariae presence in wild boar muscle samples.

The protocol of Trichinella sp. detection using artificial digestion with magnetic stirrer is described in Annex I, Chapter I of the regulation EC No. 2075/2005 (EC2005). Individual wild boar muscle samples (50 g) were separated from membranes and tendons before the sample preparation. Tap water $\left(46\right.$ to $48{ }^{\circ} \mathrm{C}$ ) was poured into $1 \mathrm{~L}$ flask and $8 \mathrm{~mL}$ of $25 \%$ hydrochloric acid and $5 \mathrm{~g}$ pepsin (1:10000 NF) were added. Muscle samples were crushed with a meat grinder until no visible pieces of muscles were present. Previously prepared digestion fluid was added to each ground muscle sample and a 5-cm-long stir bar was added. Glass was placed on the magnetic stirrer and the artificial digestion regime was at 44 to $46^{\circ} \mathrm{C}$, stirred for 30 $\min$. Afterwards, the digestion fluid was drained through a sieve $(\mathrm{d}=11 \mathrm{~cm}$, mesh size $180 \mu \mathrm{m})$ into the sedimentation funnel and the digestion fluid was allowed to stand for 30 $\mathrm{min}$. Then, $40 \mathrm{~mL}$ of the digestion fluid was quickly run off into a measuring cylinder and allowed to stand for 10 min. Supernatant $(30 \mathrm{~mL})$ was then carefully withdrawn by suction to remove the upper layers and leave a volume of not more than $10 \mathrm{~mL}$. The remaining $10 \mathrm{~mL}$ sample of sediment was poured into a Petri dish. Subsequently, the sample was examined by stereo-microscope at a 20 times magnification. In addition to standard protocol, in the present study we analyzed the remaining material in the sieve. The used sieve was carefully washed off with tap water and the resulting solution was collected in a Petri dish. The collected sample was examined by stereo-microscope at a 20 times magnification.

The protocol of Alaria sp. mesocercariae migration technique was used as described by Riehn et al. (2010) with some modification. Briefly, the $50 \mathrm{~g}$ of different wild boar muscles were selected and separated from the membranes and tendons. A rubber tube attached to a funnel was concluded with a $60 \mathrm{~mm}$ clip. Wild boar muscle was chopped with scissors approximately in $5 \times 5 \mathrm{~mm}$ pieces and placed in a sieve with a mesh size of $0.8 \mathrm{~mm}$.
The sieve was placed in a glass funnel, then rinsed with 300 $\mathrm{mL}$ of 46 to $48^{\circ} \mathrm{C}$ tap water in a way such that the meat was completely submerged in water. The suspension was settled for 30, 60 and $90 \mathrm{~min}$ at room temperature. After that, the clip was opened and $40 \mathrm{~mL}$ fluid was quickly run off into a measuring cylinder and allowed to stand for 10 min. Supernatant $(30 \mathrm{~mL})$ was then carefully withdrawn by suction to remove the upper layers and leave a volume of not more than $10 \mathrm{~mL}$. The remaining $10 \mathrm{~mL}$ sample of sediment was poured into a Petri dish. Subsequently, the sample was examined by stereo-microscope at a 20 times magnification.

All mesocercariae of A. alata were identified by morphological characteristics and counted (Möhl et al. 2009). $A$. alata mesocercariae prevalence in wild boar meat samples was calculated as defined by Bush et al. (1997) and prevalence of both methods was compared using Pearson's chi-square test and Odds Ratio (conditional maximum likelihood estimate of Odds Ratio) calculated using $\mathrm{R}$ software.

\section{Results}

In the present study 60 wild boar muscle samples were examined both with AMT and TIM. A total of 46 examined samples were found to be infected with $A$. alata mesocercariae with both methods. However, significant $\left(\chi^{2}\right.$ $=16.59 ; p<0.01)$ difference of mesocercariae prevalence was found between the methods: AMT had 4.86 (95\% CI: 2.22-10.99) times higher odds of testing positive than with TIM. The number of counted mesocercariae with AMT ranged from 1 to 61 , which equals 0.02 to 1.22 mesocercariae per one gram, while, the number of counted mesocercariae with TIM ranged from 0.02 to 0.56 mesocercariae per one gram. The mean intensity of $A$. alata mesocercariae per sample was observed to be significantly $(p<0.01)$ higher using AMT (Table 1).

Using TIM, trematodes were counted in a Petri dish and, additionally, those remaining in the sieve. In the sieve the number was 2.75 (95\% CI: 1.20-6.54) times higher odds of testing positive than following the standard protocol

Table 1. Comparison of Alaria sp. mesocercariae migration technique (AMT) and Trichinella inspection method (TIM)

\begin{tabular}{|lcc|}
\hline Parameter & TIM & AMT \\
\hline No. of analyzed samples & 60 & 60 \\
\hline No. of infested samples & 24 & 46 \\
\hline Minimal count of mesocercariae & 1 & 1 \\
\hline Maximal count of mesocercariae & 28 & 61 \\
\hline Median & 3 & 5 \\
\hline Intensity & 5 & 9.2 \\
\hline Standard deviation & 5.9 & 14.1 \\
\hline Prevalence & $40.0 \%$ & $76.7 \%$ \\
\hline (Clopper-Pearson CI 95\%) & $(27.6-53.5)$ & $(64.0-86.6)$ \\
\hline
\end{tabular}




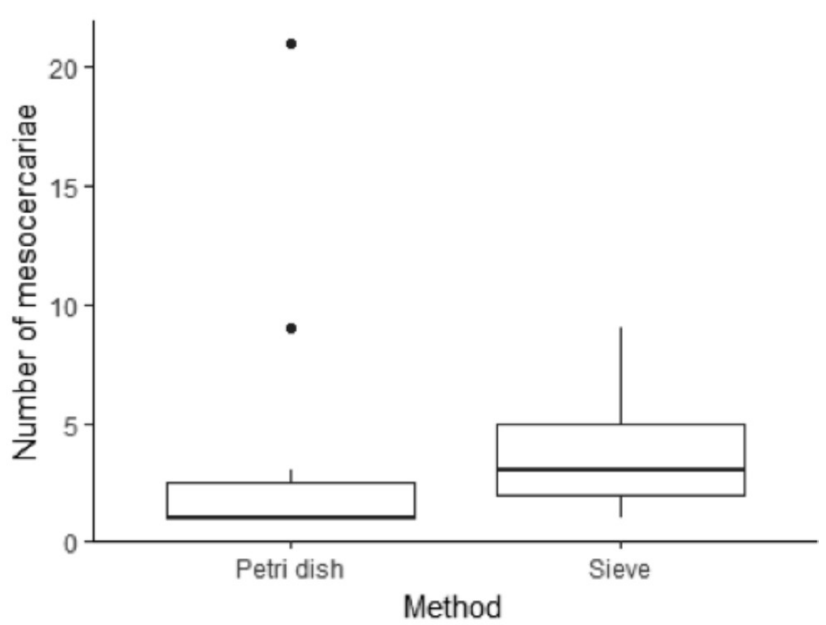

Fig. 1. The number of A. alata mesocercariae using standard protocol of Trichinella inspection method (TIM) comparing to additional mesocercariae findings using TIM in the sieve.

(Fig. 1). Overall, in 13 samples mesocercariae only in the sieve were found that went undetected by the application of standard protocol of TIM, which corresponds to $21.7 \%$ of the samples being false negatives using official Trichinella testing method. Only in one sample, mesocercariae were not found in the sieve while two mesocercariae were found in Petri dish.

Using AMT, the samples were examined after 30, 60 and $90 \mathrm{~min}$. In the first $30 \mathrm{~min}, A$. alata mesocercariae were found in 46 samples (76.7 \%, CI 95\% 64.0-86.6) samples (Table 1). In these samples, trematodes were also observed after 60 and $90 \mathrm{~min}$, however only one trematode per each sample was found (Fig. 2.).

\section{Discussion}

The present study shows that AMT is significantly better method for A. alaria mesocercariae detection compared to the TIM standard procedure. These results can be explained by the different biology of Alaria sp. and Trichinella sp., specifically different sizes of both parasites. A. alata mesocercariae reaches the size of 0.3 to $0.7 \mathrm{~mm}$ in length and 0.141 to $0.282 \mathrm{~mm}$ in width, while Trichinella sp. larvae are 0.775 to 1.050 (average 0.908 ) $\mathrm{mm}$ in length, and 0.026 to 0.042 (average 0.035 ) $\mathrm{mm}$ in maximum width (Skrjabin 1960; Sohn et al. 2000; Möhl et al. 2009). Trematodes do not generate cysts and migrate in host muscle tissue without interruption (Möhl et al. 2009; Riehn et al. 2010). However, a recent study showed that mesocercariae of $A$. alata in amphibian muscle tissues form a cyst-like structure around itself (Patrelle et al. 2015). Temporarily there is no proof that mesocercariae do not leave this cyst and the material of the cyst is unclear.

Mesocercariae prevalence studies in wild boar muscle samples from East Germany showed that false results occurred in $11.5 \%$ of the samples when TIM was used

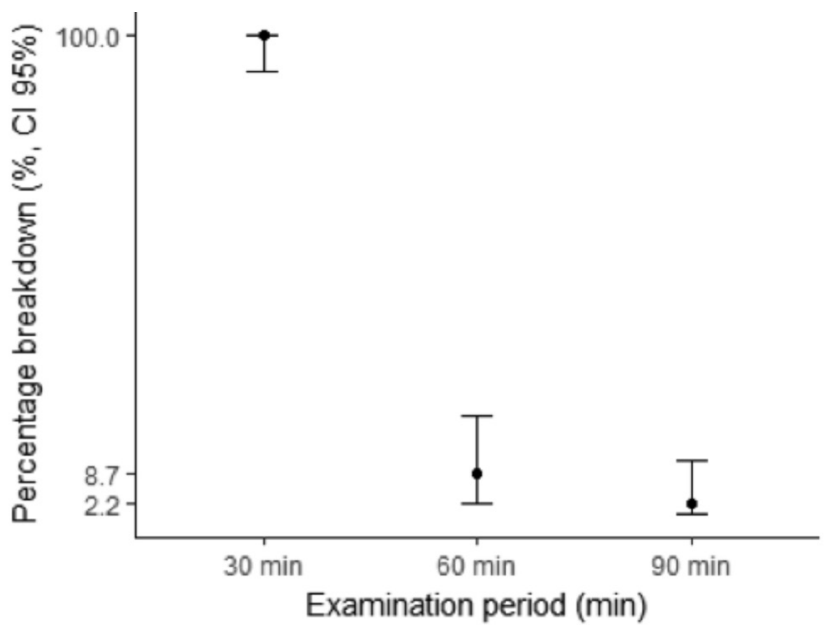

Fig. 2. The percentage of detected A. alata mesocercariae in 46 infected wild boar muscle samples using Alaria sp. mesocercariae migration technique after examination time of 30,60 and $90 \mathrm{~min}$.

(Riehn et al. 2012). In the present study, using standard protocol of TIM together with additional mesocercariae counting in the sieve, we established mesocercariae occurred significantly more frequently than found only in the Petri dish, indicating that routinely we obtain $58.4 \%$ of false results originally.

The methods for adult $A$. alaria are approbated, but detection methods for mesocercariae are still being standardized (Riehn et al. 2010). Paulsen et al. (2012) described a modification for AMT (cut musculature dipped in hot water and let stand for $45 \mathrm{~min}$ ) with the proposed time two times shorter as described previously by Riehn et al. (2010). Also for A. alata prevalence studies in frog muscle tissues, a reduced time $(30 \mathrm{~min}$ ) was used (Patrelle et al. 2015). The present study demonstrated that a reduced time (30 $\mathrm{min}$ ) ensured qualitative results (positive or negative); after 60 and $90 \mathrm{~min}$ A. alata mesocercariae were found in $5 \%$, which ensured quantitative results. A study in Germany demonstrated that in more than $95 \%$ cases when samples were let to stand for only $30 \mathrm{~min}$, there were no significant differences in the mesocercariae count compared with results from samples left to stand for $90 \mathrm{~min}$ (Riehn et al. 2010). These results correspond with the results from our study. The study in Germany showed that, only in a few cases, one mesocercariae per muscle sample was found when the sample stand for 60 to $210 \mathrm{~min}$ (Riehn et al.2010).

The present study demonstrated that cause of low mesocercariae observation using TIM was mainly associated with inappropriate mesh size of the sieve used, compared to A. alata mesocercariae size. Shortening the AMT procedure time for $30 \mathrm{~min}$ was sufficient to obtain qualitative results for $A$. alata mesocercariae in muscle samples. However, our results do not represent prevalence of A. alata mesocercariae in the whole territory of Latvia due to deficiency of data. 


\section{Acknowledgements}

The authors also thank the staff of the Institute of Food Safety, Animal Health and Environment "BIOR" for their cooperation and positive attitude towards the study.

\section{References}

Bush A.O., Lafferty K.D., Lotz J.M., Shostak A.W. 1997. Parasitology meets ecology on its own terms: Margolis et al. revisited. J. Parasitol. 83: 575-583.

Esīte Z., Bagrade G., Deksne G. 2013. Alaria alata in mammals of Latvia. Trop. Med. Int. Health 18: 226.

Galaktionov K.V., Dobrovolskij A.A. 2003. The Biology and Evolution of Trematodes. Kluwer Academic Publishers, Dordrecht.

Jakšić S., Uhitil S., Vučemilo M. 2002. Nachweis von Mesozerkarien des Saugwurms Alaria alata im Wildschweinelfleisch. $Z$. Jagdwiss. 48: 203-207

Möhl K., Große K., Hamedy A., Wüste T., Kabelitz P., Lücker E. 2009. Biology of Alaria spp. and human exposition risk to Alaria mesocercariae - a review. Parasitol. Res. 105: 1-15.

Patrelle C., Portier J., Jouet D., Delorme D., Ferté H. 2015. Prevalence and intensity of Alaria alata (Goeze, 1792) in water frogs and brown frogs in natural conditions. Parasitol. Res. 114: 4405-4412.

Paulsen P., Ehebruster J., Irsch I., Lücker E., Riehn K., Winkelmayer R., Smulders F. J. M. 2012. Finding of Alaria alata in wild boars (Sus scrofa) in eastern Austria. Eur. J. Wildl. Res. 58: 991-995.

Paulsen P., Kukla P., Bachkönig N. 2014. An update on findings of Alaria alata mesocercariae in wild boar from Austria. In: Paulsen P., Bauer A., Smulders F.J.M. (eds) Trends in Game Meat Hygiene. Wageningen Academic Publishers, pp. 203-208.

Portier J., Vallée I., Lacour S.S., Martin-Schaller R., Ferté H. Durand B. 2014. Increasing circulation of Alaria alata mesocercaria in wild boar populations of the Rhine valley, France, 2007-2011.
Vet. Parasitol. 199: 153-159.

EC 2005. Regulation EC No 2075/2005 of the European parliament and of the council of 5 December 2005 laying down specific rules on official controls for Trichinella in meat. Offic. J. EC L. 338: 60-82.

Rentería-Solis Z., Hamedy A., Michler F.U., Michler B.A., Lücker E., Stier N., Wibbelt G., Riehn K. 2013. Alaria alata mesocercariae in raccoons (Procyon lotor) in Germany. Parasitol. Res. 112: 3595-3600.

Riehn K., Hamedy A., Große K., Zeitler L., Lücker E. 2010. A novel detection method for Alaria alata mesocercariae in meat. Parasitol. Res. 107: 213-220.

Riehn K., Hamedy A., Große K., Zeitler L., Lücker E. 2011 a. Alaria alata - Nachweis, prävalenz und risikobewerung. Fleischwirtschalft. 91: 88-92.

Riehn K., Hamedy A., Alter T. 2011b. Development of a PCR approach for differentiation of Alaria spp. mesocercariae. Parasitol. Res. 108: 1327-1332.

Riehn K., Große K., Lücker E. 2011c. Nachweis des Dunker`schen Muskelegels im Rahmen der amatlichen Fleischuntersuchung mittels des modifizierten Larvenauswanderverfahrens (Alaria alata mesocercariae migration technique, AMT). J. Verbrauch. Lebensm. 6: 184.

Riehn K., Hamedy A., Große K., Wüste T., Lücker E. 2012. Alaria alata in wild boars (Sus scrofa, Linnaeus, 1758) in the eastern parts of Germany. Parasitol. Res. 111: 1857-1861.

Riehn K., Hamedy A., Saffaf J., Lücker E. 2013. First interlaboratory test for the detection of Alaria spp. mesocercariae in meat samples using the Alaria spp. mesocercariae migration technique (AMT). Parasitol. Res. 112: 2653-2660.

Sohn W.M., Kim H.M., Chung D.I., Sung T.Y. 2000. The first human case of Trichinella spiralis infection in Korea. Korean J. Parasitol. 38: 111-115.

Skrjabin K.I. 1960. Trematodes of Animals and Humans. T 18. Academy of Sciences USSR, Moscow. /in Russian/ 